\title{
Germline GATA2 variant disrupting endothelial eNOS function and angiogenesis can be restored by c-Jun/AP-1 upregulation
}

\section{Giulio Purgatorio, ${ }^{1}$ Elisa Piselli, ${ }^{1}$ Giuseppe Guglielmini,${ }^{1}$ Emanuela Falcinelli, Loredana Bury, ${ }_{1}^{1}$ Valeria Di Battista, ${ }^{2}$ Fabrizia Pellanera, ${ }^{2}$ Francesca Milano, ${ }^{2}$ Caterina Matteucci, ${ }^{2}$ Cristina Mecucci ${ }^{2 \#}$ and Paolo Gresele ${ }^{1 \#}$}

${ }^{1}$ Section of Internal and Cardiovascular Medicine, Department of Medicine and Surgery, University of Perugia and ${ }^{2} \mathrm{Hematology}$ and Bone Marrow Transplantation Unit, Department of Medicine and Surgery, University of Perugia, Perugia, Italy.

Volume 107(5):1072-1085

${ }^{\#} P G$ and $C M$ contributed equally as co-senior authors.

\section{ABSTRACT}

T ATA2 is a transcription factor with key roles in hematopoiesis. Germline GATA2 gene variants have been associated with several inherited and acquired hematologic disorders, including myelodysplastic syndromes. Among the spectrum of GATA2 deficiency-associated manifestations thrombosis has been reported in $25 \%$ of patients, but the mechanisms are unknown. GATA2 was shown to be involved in endothelial nitric oxide synthase (eNOS) regulation and vascular development. We assessed eNOS expression and angiogenesis in patients with GATA2 deficiency. Platelets and blood outgrowth endothelial cells (BOEC) from GATA2 variant carriers showed impaired $\mathrm{NO}$ production and reduction of eNOS $\mathrm{mRNA}$ and protein expression and of eNOS activity. GATA2 binding to the eNOS gene was impaired in BOEC from GATA2-deficient patients, differently from control BOEC. GATA2 deficiency BOEC showed also defective angiogenesis, which was completely restored by treatment with the NO-donor Snitroso-N-acetylpenicillamine (SNAP). Atorvastatin, but not resveratrol, largely restored eNOS expression, $\mathrm{NO}$ biosynthesis and neoangiogenesis in GATA2-deficient BOEC by a mechanism involving increased expression of the eNOS transcription factor AP-1/c-JUN, replacing GATA2 when the latter is inactive. Our results unravel a possible thrombogenic mechanism of GATA2 mutations, definitely establish the regulation of eNOS by GATA2 in endothelial cells and show that endothelial angiogenesis is strictly dependent on the eNOS/NO axis. Given the ability of atorvastatin to restore $\mathrm{NO}$ production and angiogenesis by GATA2-deficient endothelial cells, the preventive effect of atorvastatin on thrombotic events and possibly on other clinical manifestations of the syndrome related to deranged angiogenesis should be explored in patients with GATA2 deficiency in an ad hoc designed clinical trial.

\section{Introduction}

Material published in Haematologica is covered by copyright. All rights are reserved to the Ferrata Storti Foundation. Use of published material is allowed under the following terms and conditions:

https://creativecommons.org/licenses/by-nc/4.0/legalcode. Copies of published material are allowed for personal or internal use. Sharing published material for non-commercial purposes is subject to the following conditions:

https://creativecommons.org/licenses/by-nc/4.0/legalcode, sect. 3. Reproducing and sharing published material for commercial purposes is not allowed without permission in writing from the publisher.
GATA2 is a zinc finger transcription factor with key roles in the regulation of gene expression in hematopoietic cells. ${ }^{1}$ GATA2 binds consensus sequences in promoter/enhancer regions of target genes to regulate endothelial to hematopoietic transition in the embryo and to maintain the stem cell pool regulating hematopoietic stem cell (HSC) survival and self-renewal in the adult., ${ }^{2,3}$ Less than a decade ago heterozygous variants of GATA2 were first identified as the cause of four previously described hematologic syndromes, later recognized as different manifestations of a single genetic disorder. ${ }^{4 \cdot 7}$ Heterozygous germline variants in GATA2 lead to what is now referred to as GATA2 deficiency, a mutable disorder with remarkable clinical heterogeneity involving hematopoiesis, immunity and the lymphatic system. ${ }^{2,8}$ A number of non-hematological and non-infectious complications have also 
been reported in patients with GATA2 deficiency, and among these thrombosis appears to be a rather frequent occurrence. ${ }^{2,8,9}$ Different types of thrombotic events have been reported, ranging from atherothrombotic or embolic stroke to recurrent venous thromboembolism (VTE), retinal vein thrombosis or catheter-related thrombosis, amounting to $25 \%$ of patients with GATA2 deficiency suffering thrombotic episodes, half of whom with multiple events. ${ }^{2,8,10,11}$ The reason of the high incidence of thrombosis in GATA2 deficiency is unknown, but it has been suggested to be multi-factorial since patients carrying GATA2 variants often have thrombotic risk factors, including infection, malignancy, bone marrow transplantation and central venous catheters. ${ }^{2}$ GATA2 is involved in vascular development ${ }^{12,13}$ and the knockdown of GATA2 in human endothelial cells led to vascular abnormalities,$^{14}$ suggesting a role of endothelial rather than coagulation abnormalities in the pathogenesis of thrombosis associated with GATA2 deficiency. Interestingly, GATA2 was found to act as a promoter of the endothelial nitric oxide synthase gene (eNOS), the enzyme producing nitric oxide (NO), in bovine aortic endothelial cells and in the airway epithelium. ${ }^{15,16} \mathrm{NO}$ plays a crucial role in the cardiovascular system acting as a powerful antithrombotic agent through its vaso-dilatatory, platelet inhibitory and anti atherosclerotic activities ${ }^{17-19}$ and recent data show that its deficiency is associated with enhanced risk of VTE too. ${ }^{20,21}$ However, no studies so far have explored NO production in patients with GATA2 deficiency.

Here we show for the first time that platelets and endothelial cells from patients with GATA2 deficiency due to the R398W GATA2 variant exhibit impaired NO production and defective angiogenesis which can largely be corrected by pharmacologically-induced recovery of $e$ NOS mRNA expression.

\section{Methods}

\section{Germline GATA2 mutation patients}

The proband (II2) was a 22 years old girl with mild anemia, reduction of monocytes, B and NK cells, recurrent otitis and papilloma virus infections, compatible with the MonoMac syndrome. ${ }^{4}$ Her mother (I1, 65 years old) and her sister (II1, 30 years old) did not show any clinical phenotype, and her father (I2, 66 years old) had a past clinical history of stroke and venous thrombosis. The father had suffered a first ischemic stroke at the age of 59, with no evidence of a cardioembolic source, and a recurrent ischemic stroke at the age of 66 while on aspirin. He later developed an unprovoked subclavian vein thrombosis and was then put under permanent oral anticoagulation with a direct oral anti-Xa drug. A thorough assessment for inherited or acquired thrombophilic conditions was negative.

The study was approved by Comitato Universitario di Bioetica, University of Perugia (date of approval: 01/07/2019; approval number: 2019-21). The study was conducted according to the Declaration of Helsinki. Informed consent was obtained from the proband and all family members.

\section{Blood outgrowth endothelial cells}

Blood outgrowth endothelial cells (BOEC) were isolated from peripheral blood of the proband and her family and from age- and sex-matched healthy controls and cultured as previously described. ${ }^{22,23}$ For details see the Online Supplementary Appendix.

\section{Chromatin immunoprecipitation}

Chromatin immunoprecipitation (ChiP) assay was performed using the SimpleChiP ${ }^{\circledR}$ enzymatic Chromatin IP Kit \#9002 (Cell Signaling, Danver, MA, USA), according to the manufacturer's instructions. ${ }^{24,25}$ Purified DNA was used as template for quantitative polymerase chain reaction (qPCR), using primers amplifying the eNOS promoter regions that are recognized by the AP-1 (forward: CTCAGCCCTAGTCTCTCTGC; revere: GGTTCTTGGGGATAGAGGCC) and GATA2 (forward: GGTGCCACATCACAGAAGGA; reverse: CACAATGGGACAGGAACAAGC) transcription factors, as previously described ${ }^{15}$. For details see the Online Supplemental Appendix.

\section{Protein expression: western blotting}

Proteins were extracted from BOEC, quantified using the Bradford method, and Western blotting was carried out as described. ${ }^{26,27}$ For details see the Online Supplementary Appendix.

\section{Immunofluorescence analysis of GATA2}

GATA2 distribution was evaluated by immunofluorescence analysis with confocal microscopy, as previously described ${ }^{28}$ For details see the Online Supplementary Appendix.

\section{Nitric oxide production}

$\mathrm{NO}$ generation in BOEC and platelets was studied by flow cytometry using a specific fluorescent probe (4-amino-5-methylamino-2',7'-Difluorofluorescein diacetate, DAF-FM diacetate, Invitrogen). BOEC were stimulated with acetylcholine $10 \mu \mathrm{M}$ or acetylcholine $10 \mu \mathrm{M}$ plus $N^{5}$-(1-Iminoethyl)-L-ornithine dihydrochloride (L-NIO), a NOS inhibitor ${ }^{29} 100 \mu \mathrm{M}$. Platelets were stimulated with type I collagen (Mascia Brunelli, Milan, Italy) at increasing concentrations $(1-10 \mu \mathrm{g} / \mathrm{mL})$ and $\mathrm{NO}$-generated fluorescence was analyzed as previously described. ${ }^{30}$ For details see the Online Supplementary Appendix.

\section{eNOS activity assay}

eNOS activity was measured by assessing the enzymatic conversion of $\left(\mathrm{H}^{3}\right) \mathrm{L}$-arginine to $\left(\mathrm{H}^{3}\right) \mathrm{L}$-citrulline. A standard curve with increasing $\left(\mathrm{H}^{3}\right) \mathrm{L}$-arginine concentrations was built for each assay. ${ }^{31}$ For details see the Online Supplementary Appendix.

\section{Treatment of blood outgrowth endothelial cells with eNOS inducers}

BOEC from healthy controls and from GATA2-deficient patients at passage 5 were seeded in 24 -well plates $\left(150 \times 10^{3}\right.$ cells/well) in serum-free EBM2 medium. Cells were incubated with atorvastatin at a concentration of $50 \mu \mathrm{M},{ }^{32}$ or with resveratrol $40 \mu \mathrm{M},{ }^{33}$ or with their vehicle (dimethyl sulfoxide [DMSO]) for 24 hours $(\mathrm{h})$ in serum-free medium. DMSO final concentration never exceeded $0.5 \%$.

\section{In vitro Matrigel angiogenesis assay}

Angiogenesis was estimated by measuring total tube length and by counting tubule number and branching points, as previously described $^{34}$ For details see the Online Supplementary Appendix.

\section{Statistical analysis}

Data are presented as means \pm standard error of the mean. The $t$-test for unpaired data was used to analyze results with a significant difference set at $P<0.05$. For details see the Online Supplementary Appendix.

Further details on materials and methods are available in the Online Supplementary Appendix. 


\section{Results}

\section{Variant identification and blood outgrowth endothelial cells characterization}

The proband showed a heterozygous c.1192 C>T variant in GATA2 causing an arginine to tryptophan substitution (p.R398W). This is a known mutation affecting the zinc finger 2 (ZF2) domain of GATA2 and has been first reported as a recurrent missense variant in patients with the autosomal dominant monocytopenia and mycobacterial avium complex infection syndrome (MonoMAC). ${ }^{4}$ The same heterozygous variant was found in the father and sister, who were also both affected by mild monocytopenia and reduction of B and NK cells, although to a lesser extent compared to the proband (data not shown), but not in the mother (Figure 1A). BOEC derived from GATA2-mutated patients and healthy controls showed all the typical endothelial surface markers (CD31, CD146 and CD309) (Online Supplementary Figure S1A), and expressed von Willebrand factor (Online Supplementary Figure S1AB). No difference was observed between healthy control and GATA2-mutated BOEC for viability, assessed by the fluorescein diacetate/propidium iodide (FDA/PI) assay (Online Supplementary Figure S2A) or by annexin-V/PI staining (Online Supplementary Figure S2B). Proliferation of BOEC derived from GATA2-mutated patients, assessed by bromodeoxyuridine (BrdU) incorporation, did not differ from that of healthy control BOEC (Online Supplementary Figure S3). Cells incubated with BrdU vehicle (EBM2 medium) were used as negative control.

\section{GATA2 expression and subcellular distribution}

Real time PCR did not show differences in the expression of GATA2 mRNA between BOEC from healthy controls and those from the proband and familial carriers (Figure 1B). Moreover, there was no evidence of GATA2 protein decrease compared to controls (Figure 1C). Confocal microscopy analysis of BOEC from healthy controls and

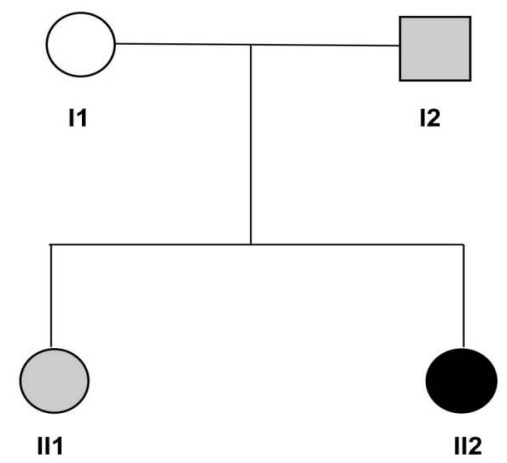

C
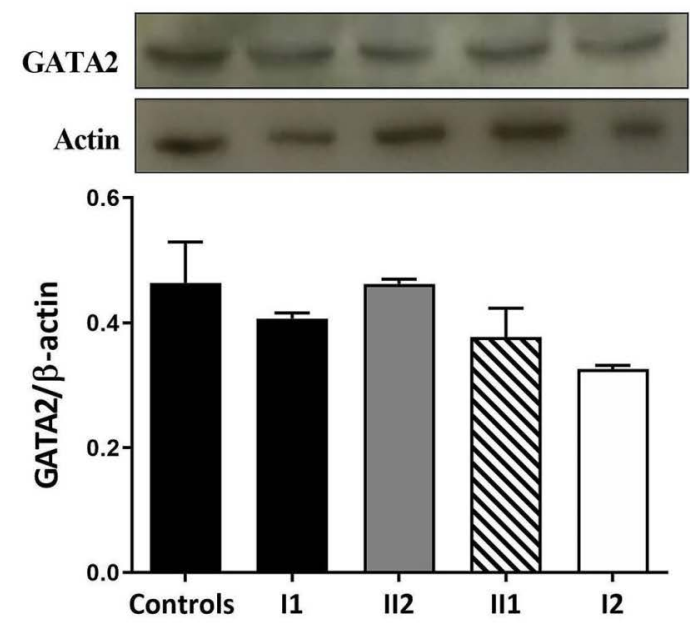

B

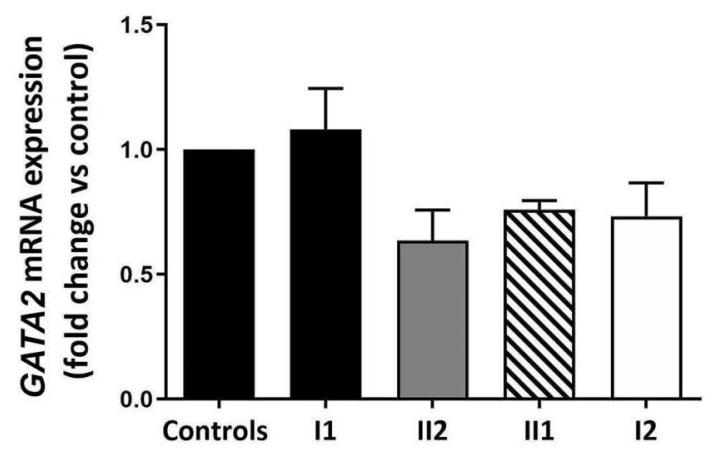

D

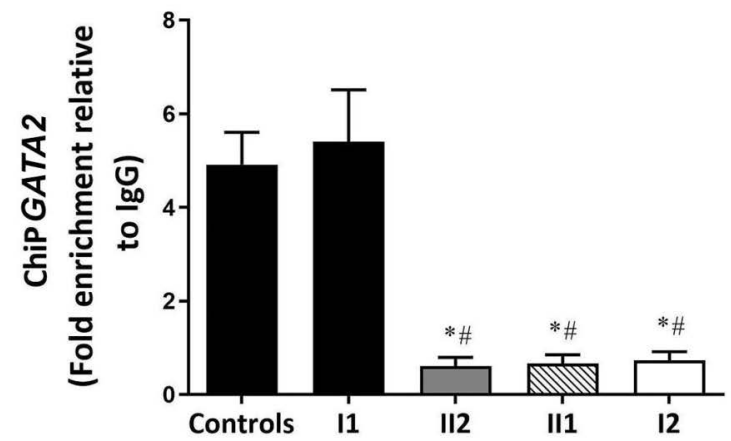

Figure 1. Normal GATA2 mRNA and protein expression and impaired GATA2 binding to DNA in blood outgrowth endothelial cells from GATA2-deficient patients. (A) Pedigree showing the family with p.R398W GATA2 variant. Square denotes male and circle females. In gray the father (I2) and the sister (II1) asymptomatic carriers; the black circle indicates the proband (II2) symptomatic carrier. The white circle indicates the wild type mother (I1). (B) Real time polymerase chain reaction (PCR) of GATA2 mRNA expression in blood outgrowth endothelial cells (BOEC) from healthy controls, the unaffected family member (I1), and the GATA2-deficient family members. Expression of GATA2 mRNA is reported as fold change versus control BOEC normalized to a housekeeping mRNA (GAPDH). Values represent mean \pm standard error of the mean (SEM) of 6 repeated measures from 6 controls and 3 different preparations from the GATA2-deficient family members (one-way ANOVA followed by Dunnett's multiple comparison test). (C) Western blotting of GATA2 protein in BOEC from healthy controls, the unaffected family member (I1) and the GATA2-deficient family members. Actin was used as loading control. Optical densitometric analysis was performed using Image J software and results are expressed in arbitrary units. Values represent mean \pm SEM of 8 repeated measures from 6 controls and 3 different preparations from the GATA2-deficient family members (one-way ANOVA followed by Dunnett's multiple comparison test). (D) Chromatin immunoprecipitation (ChiP) quantitative PCR using primers amplifying the endothelial nitric oxide synthase gene (eNOS) promoter regions that are recognized by GATA2 performed using BOEC from healthy controls, the unaffected family member (I1) and the GATA2deficient family members. Aspecific binding of chromatin was excluded using immunoglobulin $\mathrm{G}$ (IgG)-immunoprecipitated chromatin as a negative control. Values represent mean \pm SEM of 6 repeated measures from 6 controls and 3 different preparations from the GATA2 deficiency family members ( ${ }^{2}<0.001$ vs. controls, $\# P<0.001$ vs. I1; one-way ANOVA followed by Dunnett's multiple comparison test). Data are shown as fold change over lgG ( $\mathrm{n}=3$ ). 
from GATA2-deficient patients showed the same subcellular distribution of GATA2, both in the nucleus and cytoplasm, as shown by green fluorescent protein reporter expression, in agreement with previous data ${ }^{14,35}$ (Online Supplementary Figure S4).

\section{GATA2-binding to the eNOS gene and eNOS expression are impaired in GATA2 deficiency patients}

ChIP-qPCR of GATA2-bound chromatin showed that while GATA2 interacts directly with the human $e N O S$ promoter in BOEC from healthy controls and from the unaffected family member (I1), this interaction is significantly reduced in all GATA2-mutated patients (Figure 1D). The eNOS (205 bp) signal was found in INPUT chromatin and in GATA2-immunoprecipitated chromatin from BOEC from healthy controls, while no eNOS DNA band was observed in GATA2-immunoprecipitated chromatin from BOEC from the GATA2-mutated patients (Online Supplementary Figure S5). In fact, patients with GATA2 deficiency showed a significant and striking reduction of eNOS mRNA and eNOS protein, compared to healthy controls (Figure 2A and B).

\section{Silencing GATA2 reduces eNOS expression in control endothelial cells}

Transfection of healthy control BOEC with GATA2-targeted double-stranded small interfering RNA (siRNA) induced a significant reduction of GATA2 mRNA, attaining almost total suppression with the combined treatment with three different siRNA (Figure 2C). GATA2 protein expression was also significantly suppressed by GATA2-targeted siRNA, starting $36 \mathrm{~h}$ after transfection and maximally at 48 $\mathrm{h}$ (Figure 2D). Thirtysix h after transfection, when GATA2 protein was strikingly decreased, a significant reduction of eNOS mRNA was observed (Figure 2E), while complete suppression of eNOS protein expression was evident $48 \mathrm{~h}$ after GATA2-targeted siRNA transfection (Figure 2F). Treatment of healthy control BOEC with siRNA GATA2 or scramble siRNA (NC) did not affect cell viability (Online Supplementary Figure S2A and B).

\section{Platelets and blood outgrowth endothelial cells from GATA2 deficiency patients show impaired nitric oxide production}

Platelets and BOEC from patients with GATA2 deficiency showed defective NO production, as assessed by the diaminofluorescein-2 diacetate (DAF) fluorescence assay, compared with healthy controls. In particular, while platelets from healthy controls stimulated with increasing concentrations of collagen showed a dose-dependent rise of DAF-fluorescence, platelets from the GATA2-mutanted subjects did not. Collagen-triggered DAF fluorescence in platelets from GATA2-mutanted patients was significantly lower than that in platelets from healthy controls, at all concentrations of collagen used (Figure 3A). Similarly, BOEC from healthy controls showed a striking increase of DAF-fluorescence upon stimulation with acetylcholine (10 $\mu \mathrm{M})$, while BOEC from GATA2 deficiency subjects showed a significantly lower increase of DAF fluorescence. Acetylcholine-triggered DAF fluorescence in BOEC was completely abolished by preincubation with the eNOS inhibitor L-NIO (Figure 3B). The impairment of eNOS activity in BOEC from patients with GATA2 deficiency subjects was confirmed by the significantly reduced conversion of $\left(\mathrm{H}^{3}\right) \mathrm{L}$-arginine to $\left(\mathrm{H}^{3}\right) \mathrm{L}$-citrulline in resting and acetylcholine-stimulated cells. Here too L-NIO suppressed $\mathrm{H}^{3}$-L-citrulline generation (Figure $3 \mathrm{C}$ ). Moreover, the amount of the $\mathrm{NO}$ metabolites $\left[\mathrm{NO}_{2}-\mathrm{NO}_{3}-\right.$ ](NOx) released in the supernatant by acetylcholine-stimulated BOEC from GATA2-deficient subjects was significantly reduced compared to BOEC from healthy subjects (Figure 3D). Finally, levels of NOx in circulating blood from patients with GATA2 deficiency were also significantly reduced compared to healthy controls (Online Supplementary Figure S6). Acetylcholine-triggered NO release by control BOEC transfected with GATA2-targeted siRNA, as assessed by the DAF assay, was strikingly reduced compared with BOEC transfected with scramble siRNA (Figure 3E).

\section{GATA2 and eNOS are required for angiogenesis}

BOEC from patients with GATA2 deficiency showed impaired angiogenesis in a tube formation assay compared with BOEC from healthy controls. Indeed tube length, branching points and the number of tubes were significantly reduced (Figure 4). In order to confirm the role of GATA2 and eNOS in angiogenesis, microvessel sprouting was evaluated in GATA2-silenced control BOEC. Tube formation was normal $36 \mathrm{~h}$ after GATA2 silencing, when GATA2 protein is suppressed but eNOS protein is not yet reduced (Online Supplementary Figure S7), while $48 \mathrm{~h}$ after transfection, when both GATA2 and eNOS were suppressed, angiogenesis was impaired with alterations similar to those found in BOEC from patients with GATA2 deficiency (Figure 4). In order to further clarify whether impaired eNOS expression, and not GATA2 suppression, was responsible of altered angiogenesis in patients with GATA2 deficiency, we treated their BOEC with the NO donor SNAP (100 $\mu \mathrm{M})$ for $24 \mathrm{~h}$ and complete restoration of angiogenesis was observed. Similarly, treatment of GATA2-silenced control BOEC with the NO donor SNAP $(100 \mu \mathrm{M})$ restored angiogenesis by significantly increasing tube number, branching points and tube lenght (Figure 4). Concordantly, treatment of control BOEC with the eNOS inhibitor L-NIO $(100 \mu \mathrm{M})$ impaired tube formation (Online Supplementary Figure S8).

\section{The eNOS inducer atorvastatin restores eNOS expression and nitric oxide production in blood outgrowth endothelial cells from GATA2-deficient patients}

Incubation with atorvastatin of BOEC from GATA2-deficient patients and from healthy controls for $24 \mathrm{~h}$ significantly increased eNOS mRNA expression compared with vehicle. On the contrary, treatment with resveratrol enhanced mRNA expression in healthy control BOEC but not in GATA2-mutated BOEC (Figure 5A). These data were confirmed by western blotting of eNOS protein, showing a significant increase of eNOS protein expression with atorvastatin in healthy control and GATA2-mutated BOEC but only in healthy control BOEC resveratrol (Online Supplementary Figure $S 9 A$ and B). BOEC derived from the unaffected family member (I1) showed the same behavior of healthy control BOEC (Online Supplementary Figure S9C). Increased eNOS expression induced by preincubation with atorvastatin was associated with enhanced $\mathrm{NO}$ production in both control and GATA2-deficient BOEC, as assessed by the DAF assay and by the measurement of NOx in cell supernatant (Figure 5B). On the contrary, preincubation with resveratrol enhanced the release of NO from control 


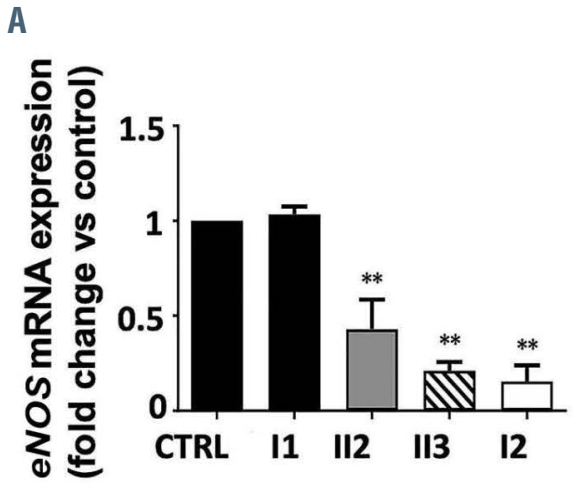

C

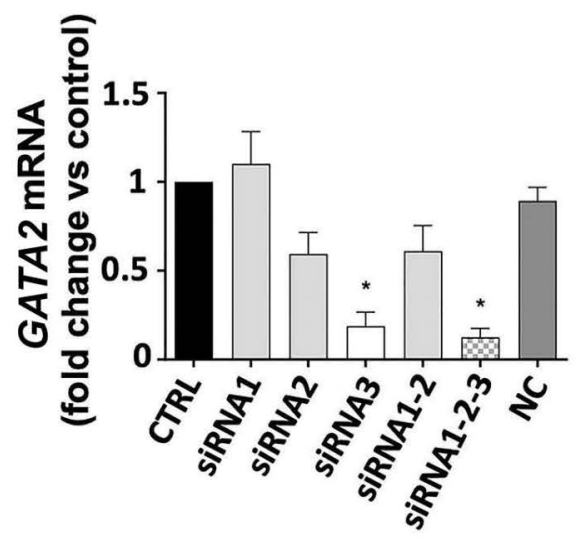

E

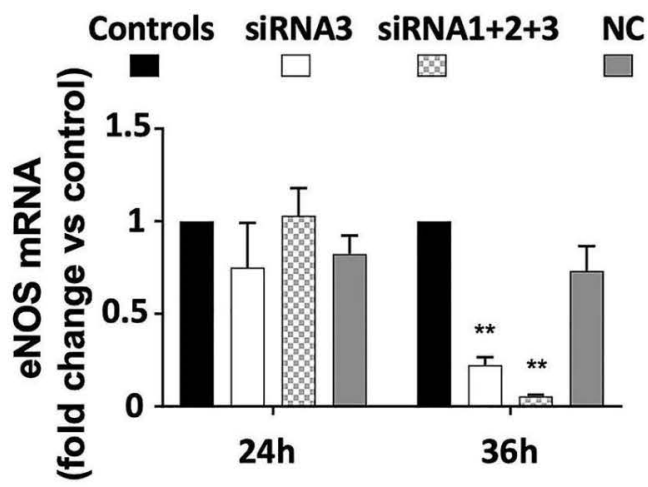

B
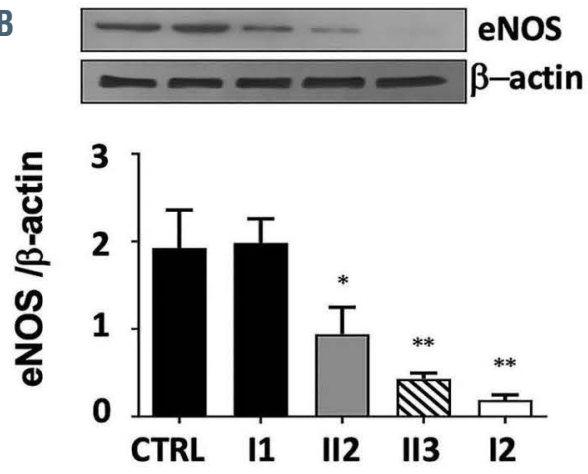

D
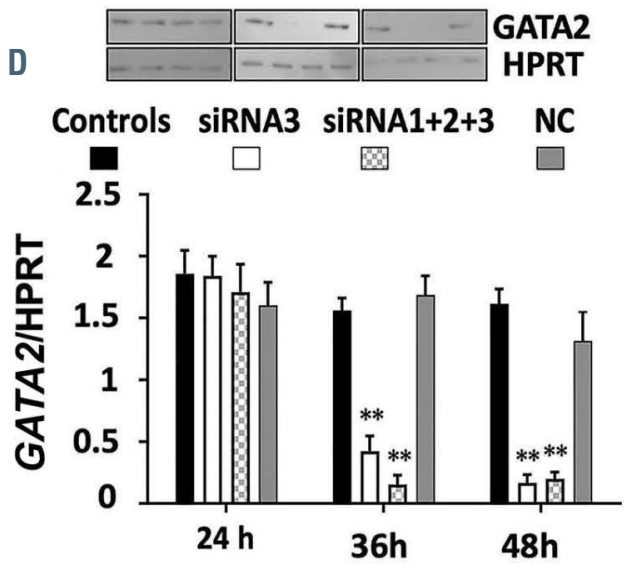

F
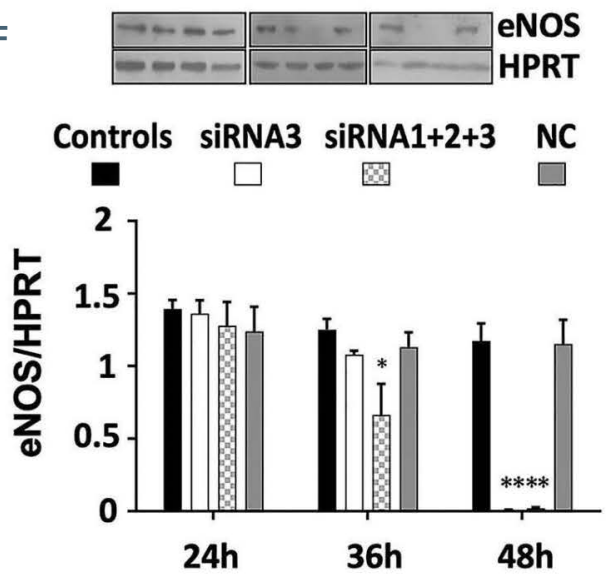

Figure 2. Impaired GATA2 activity and expression alter eNOS mRNA and protein expression. (A) Real time polymerase chain reaction (PCR) of the endothelial nitric oxide synthase gene (eNOS) mRNA expression in blood outgrowth endothelial cells (BOEC) from healthy controls, the unaffected family member (I1) and the GATA2-deficient family members. eNOS mRNA expression is reported as fold change versus healthy control BOEC normalized to a housekeeping mRNA (GAPDH). Values represent mean \pm standard error of the mean (SEM) of 8 repeated measures from 6 controls and 3 different preparations from the family members (**P<0.001 vs. controls; one-way ANOVA followed by Dunnett's multiple comparison test). (B) Western blotting of eNOS protein in BOEC from healthy controls, the unaffected family member (I1) and the GATA2 deficiency family members. Actin was used as loading control. Optical densitometric analysis was performed using ImageJ software and results are expressed in arbitrary units. Values represent mean \pm SEM of 6 repeated measures from 6 controls and 3 different preparations from the patients (one-way ANOVA followed by Dunnett's multiple comparison test, ${ }^{*} P<0.005, * * P<0.001$ vs. controls). (C) Real time PCR of GATA2-coding mRNA extracted from BOEC after incubation with three different small interfering RNA (siRNA) directed against GATA2 $(25 \mathrm{nM})$ and their combinations. GATA2 expression was normalized to that of GAPDH. Values represent mean \pm SEM of 4 repeated measures ( $* P<0.05$ vs. controls, one-way ANOVA followed by Bonferroni's multiple comparison test). (D) Western blotting of GATA2 protein extracted from BOEC after incubation with siRNA directed against GATA2 (25 nM) for 24-48 hours (h). HPRT was used as a loading control. Optical densitometric analysis was performed using ImageJ software and results are expressed in arbitrary units. Values represent mean \pm SEM of 4 repeated measures (**P<0.001 vs. untreated BOEC, two-way ANOVA followed by Dunnett's multiple comparison test). (E) Expression of eNOS mRNA after $24 \mathrm{~h}$ and $36 \mathrm{~h}$ of incubation of control BOEC with siRNA directed against GATA2 $(25 \mathrm{nM})$ measured by real time PCR. The expression of eNOS is reported as fold change versus untreated BOEC and normalized to a housekeeping mRNA (GAPDH). Values are means \pm SEM of 6 different transfection experiments ( $* * P<0.005$ vs. control BOEC, one way-ANOVA followed by Bonferroni's multiple comparison test). (F) Western blotting of eNOS protein expression in BOEC treated with siRNA directed against GATA2 (25 nM) for 24,36 and $48 \mathrm{~h}$. HPRT was used as loading control. Optical densitometric analysis was performed using ImageJ software and results are expressed in arbitrary units. Values are mean \pm SEM of 6 different transfection experiments ( $* * P<0.001$ vs. control BOEC, two-way ANOVA followed by Dunnett's multiple comparison test). 
PLATELETS
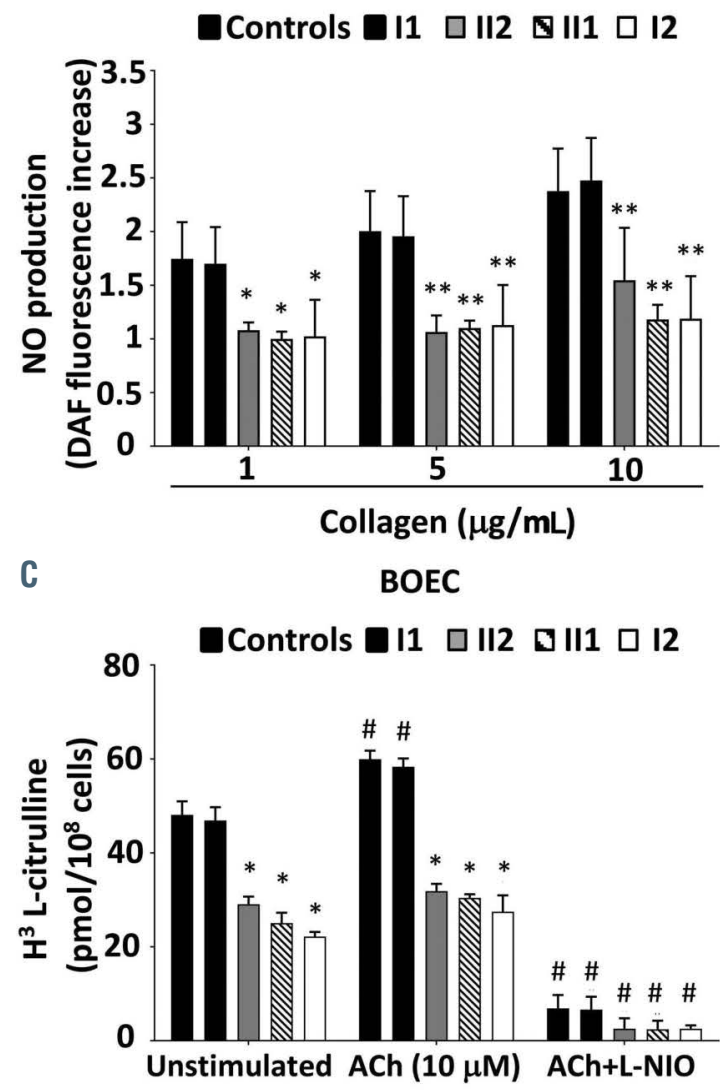

BOEC

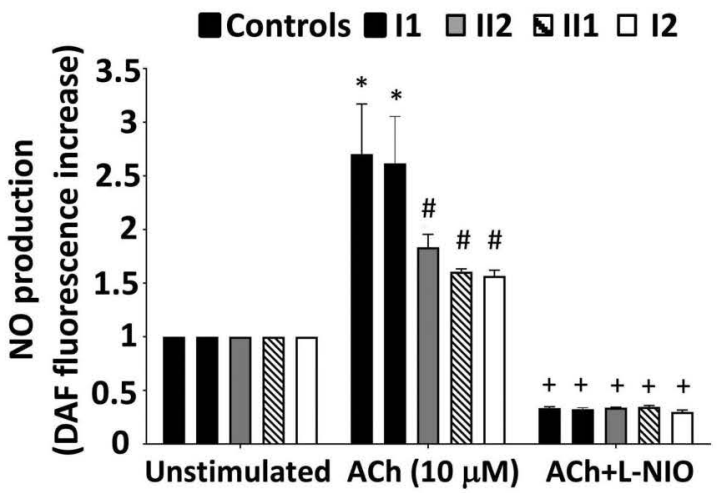

D

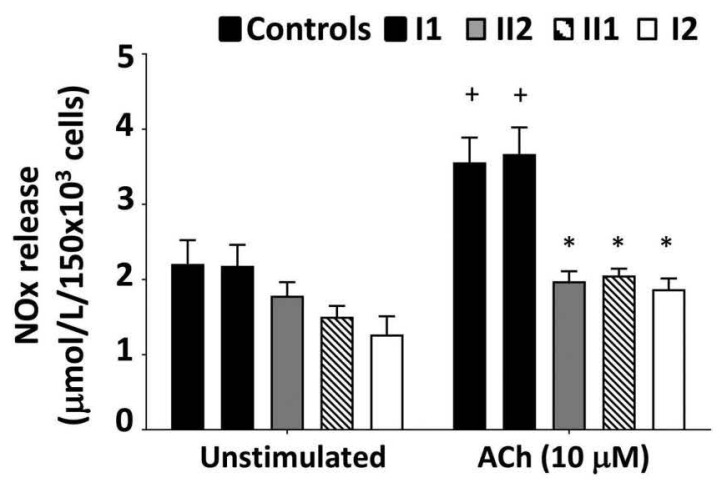

E

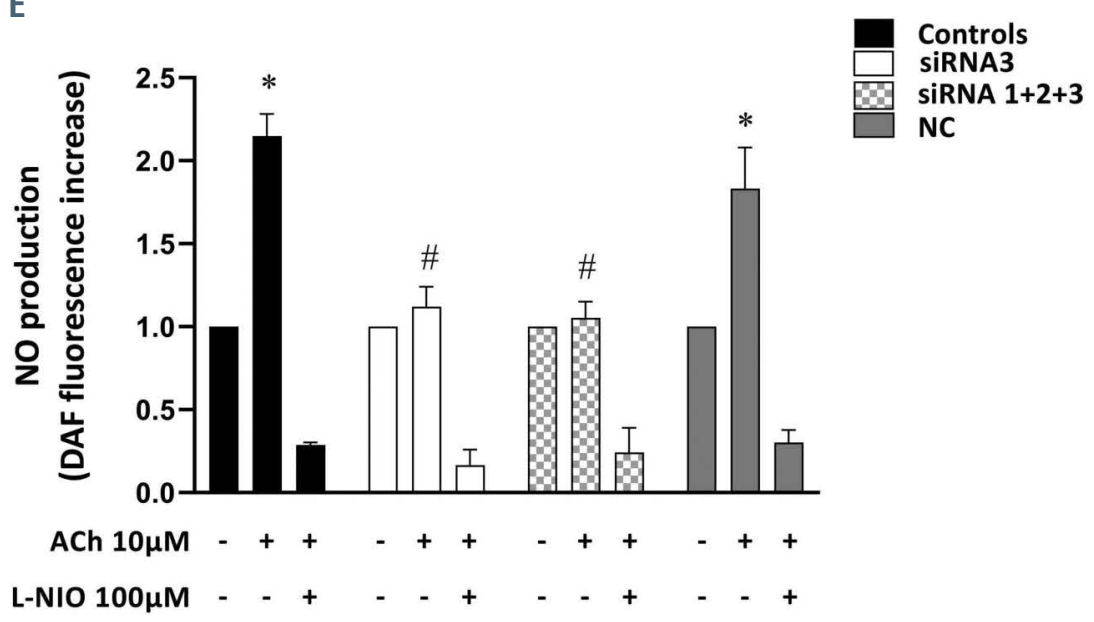

Figure 3. Impaired nitric oxide production from platelets and blood outgrowth endothelial cells from GATA2-mutated patients. (A) 4-amino-5-methylamino-2',7'-difluorofluorescein (DAF-FM)-loaded platelets from healthy controls, the unaffected family member (I1) and the GATA2-deficient family members were stimulated with collagen $(1-10 \mathrm{ug} / \mathrm{mL}$ ) and DAF-FM fluorescence intensity was recorded. Values are means \pm standard error of the mean (SEM) of 6 repeated measures $(* \star P<0.001$ vs. controls; two-way ANOVA followed by Tukey's multiple comparison test). (B) DAF-FM-loaded blood outgrowth endothelial cells (BOEC) from healthy controls, the unaffected family member (I1) and the GATA2-deficient family members were stimulated with acetylcholine (ACh) $10 \mu \mathrm{M}$ or ACh10 $\mu \mathrm{M}$ plus L-NIO $100 \mu \mathrm{M}$. Values are means \pm SEM of 6 repeated measures ( ${ }^{*} P<0.0001$ vs. baseline, ${ }^{*} P<0.005$ vs. control, ${ }^{+} P<0.005$ vs. ACh $10 \mu \mathrm{M}$; two-way ANOVA followed by Tukey's multiple comparison test). (C) Endothelial nitric oxide synthase gene (eNOS) activity $\left({ }^{3} \mathrm{H}\right) \mathrm{L}$-Citrulline production) of acetylcholine- and ACh plus L-NIO-stimulated BOEC from healthy controls, the unaffected family member (I1) and the GATA2-deficient family members. Values are means \pm SEM of 6 repeated measures ( ${ }^{2} P<0.001$ vs. controls, ${ }^{\#} P<0.001$ vs. unstimulated, two-way ANOVA followed by Tukey's multiple comparison test). (D) Nitrite and nitrate (NOx) released in the supernatant from healthy controls, the unaffected family member (I1) and the GATA2-deficient family members under resting conditions and after stimulation with ACh $10 \mu \mathrm{M}$ for 30 minutes. Concentration is expressed in $\mu \mathrm{M} / \mathrm{L} / 150 \times 10^{3}$ cells. Values are means \pm SEM of 6 repeated measures $(+P<0.001$ vs. Baseline, * $P<0.001$ vs. controls; two-way ANOVA followed by Tukey's multiple comparison test). (E) DAF-FM-loaded BOEC silenced with small interfering RNA (siRNA) directed against GATA2 or scramble siRNA were stimulated with acetylcholine $10 \mu \mathrm{M}$ or acetylcholine $10 \mu \mathrm{M}$ plus L-NIO $100 \mu \mathrm{M}$ and fluorescence was recorded. Values are means \pm SEM of 6 repeated measures ( ${ }^{*} P<0.001$ vs. unstimulated, ${ }^{*} P<0.001$ vs. controls and scramble, two-way ANOVA followed by Tukey's multiple comparison test). 

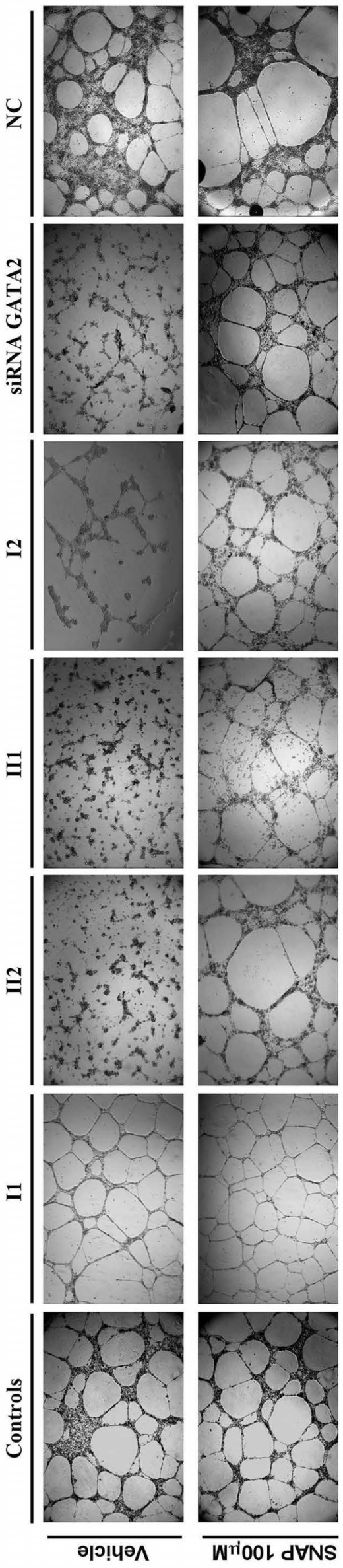

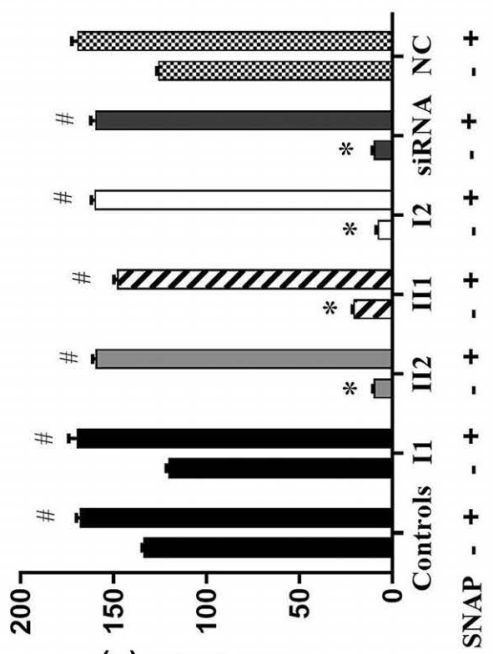

(u) səqnł ןełol

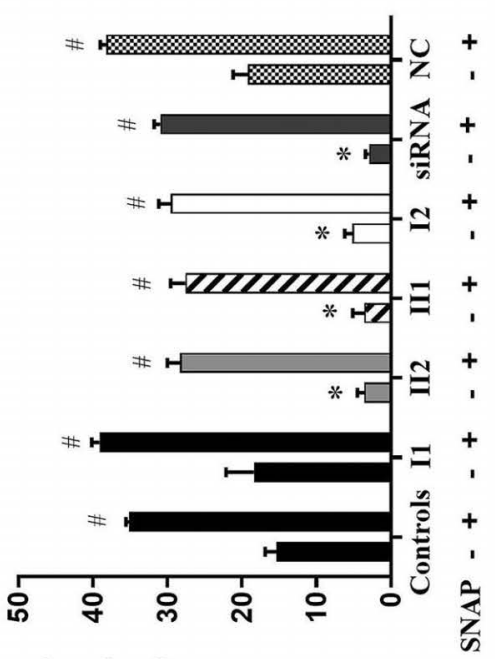

(uس) ł46uә әqnł ןeł०।

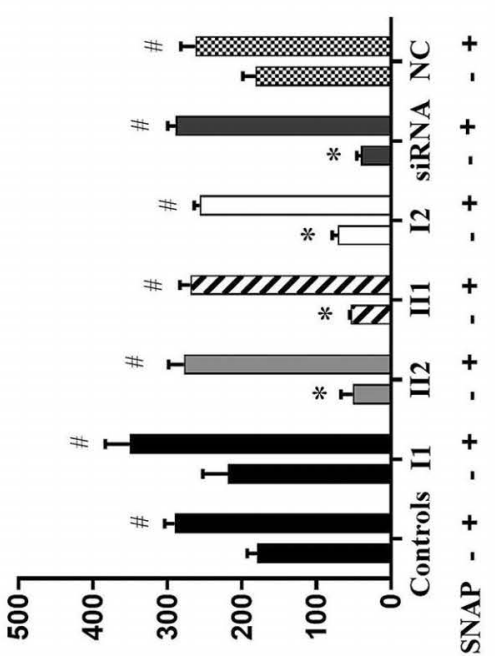

(u) słu!od 6u!ysuesg

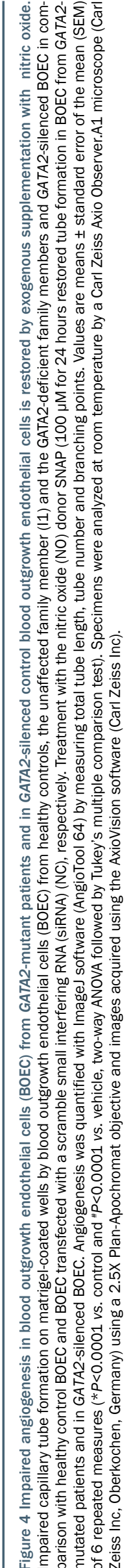


A Vehicle $\square$ Atorva $\square$ Resv
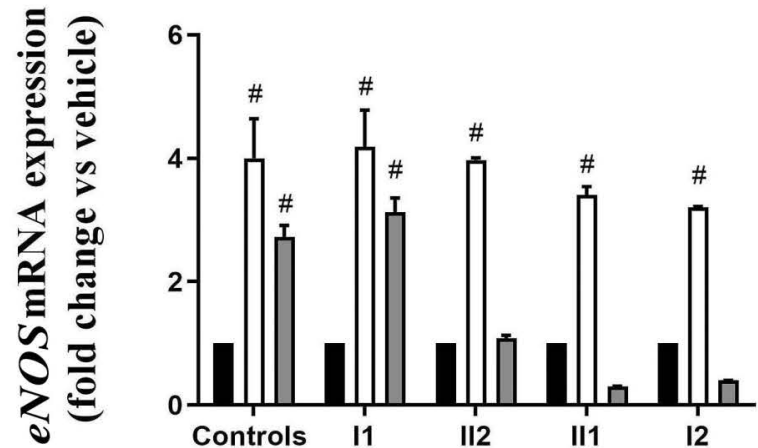

B

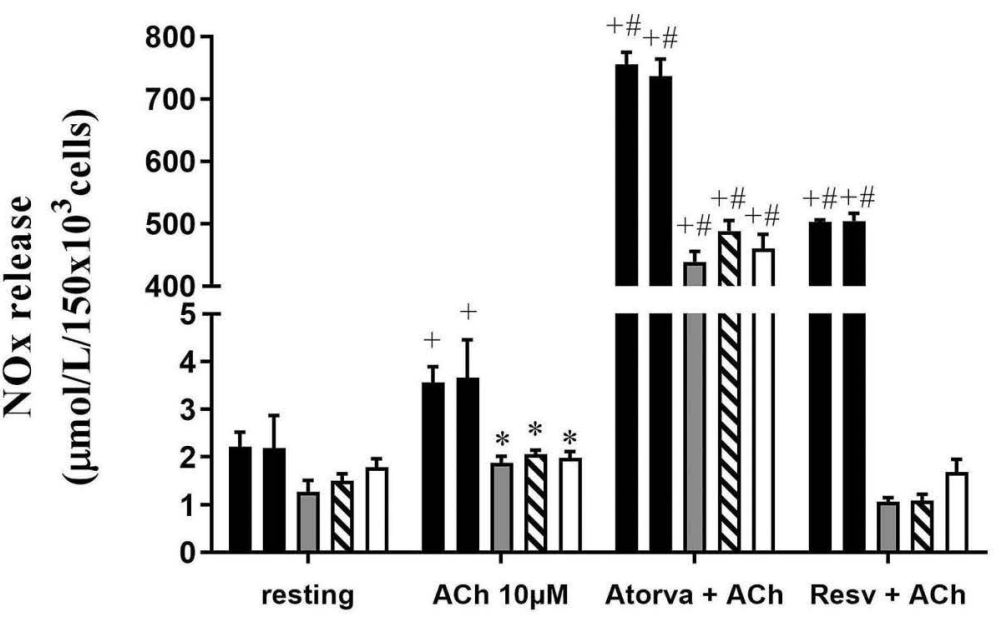

C

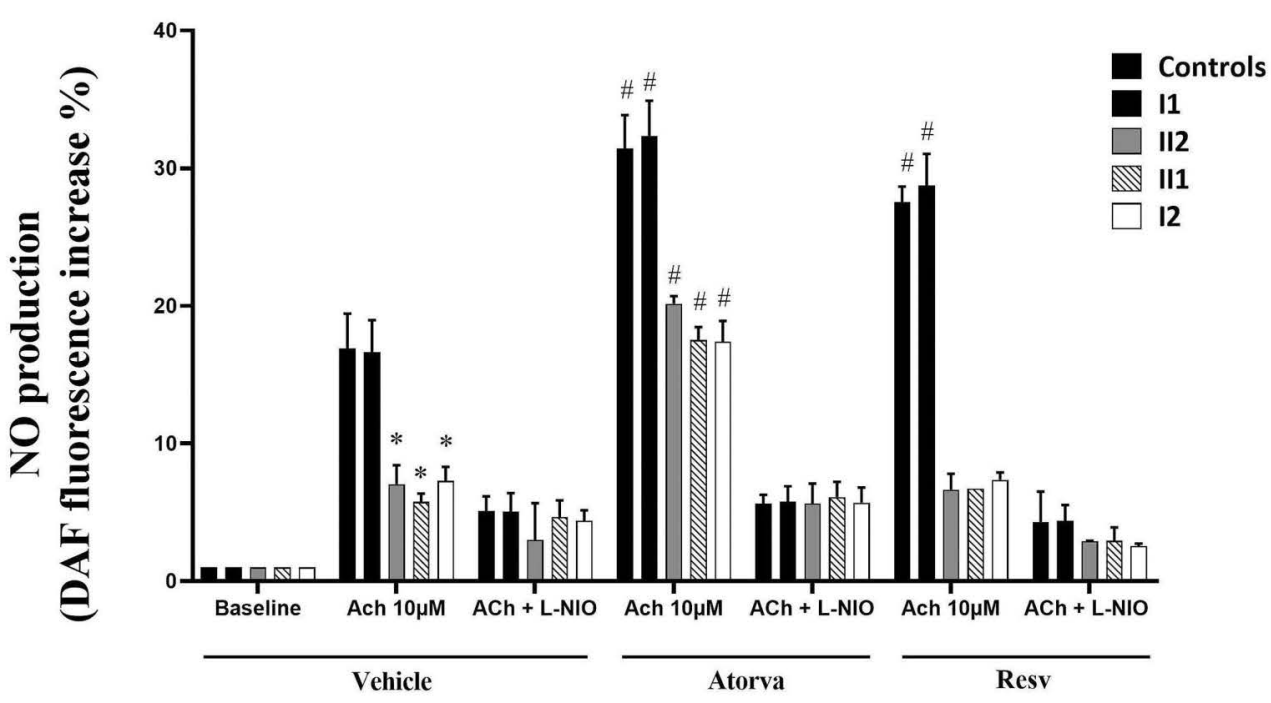

Figure 5. Atorvastatin, but not resveratrol, increases eNOS expression and nitric oxide production of blood outgrowth endothelial cells from GATA2-mutant patients. (A) Real time polymerase chain reaction (PCR) of endothelial nitric oxide synthase gene (eNOS)-coding mRNA of blood outgrowth endothelial cells (BOEC) from healthy controls, the unaffected family member (I1) and the GATA2-deficient family members after preincubation with atorvastatin (50 $\mu \mathrm{M})$ or resveratrol (40 $\mu \mathrm{M})$ for 24 hours. The expression of eNOS mRNA is reported as fold change versus vehicle and normalized to a housekeeping mRNA (GAPDH). Values are means \pm standard error of the mean (SEM) of 4 repeated measures ( $P<0.001$ vs. vehicle,two-way ANOVA followed by Tukey's multiple comparison test). (B) Nitrite and nitrate released (NOx) in the supernatant of BOEC from healthy controls, the unaffected family member (I1) and the GATA2-deficient family members after incubation with atorvastatin $(50 \mu \mathrm{M})$ and resveratrol $(40 \mu \mathrm{M})$ for $24 \mathrm{~h}$. Values are means $\pm \mathrm{SEM}$ of 4 repeated measures $\left({ }^{+} P<0.0001\right.$ vs. resting, ${ }^{*} P<0.001$ vs. control, ${ }^{*} P<0.0001$ $v s$. acetylcholine (ACh), two-way ANOVA followed by Tukey's multiple comparison test). (C) Nitric oxide (NO) production by BOEC from healthy controls, the unaffected family member (I1) and the GATA2 deficiency family members. 4-amino-5-methylamino-2',7'-difluorofluorescein (DAF-FM)-loaded BOEC, after treatment with atorvastatin $(50 \mu \mathrm{M})$ and resveratrol $(40 \mu \mathrm{M})$ for $24 \mathrm{~h}$, were stimulated with ACh $10 \mu \mathrm{M}$ or ACh $10 \mu \mathrm{M}$ plus L-NIO $100 \mu \mathrm{M}$ and fluorescence was recorded. Values are means \pm SEM of 4 repeated measures ( ${ }^{*} P<0.001$ vs. control, ${ }^{*} P<0.001$ vs. untreated, two-way ANOVA followed by Tukey's multiple comparison test). 
A
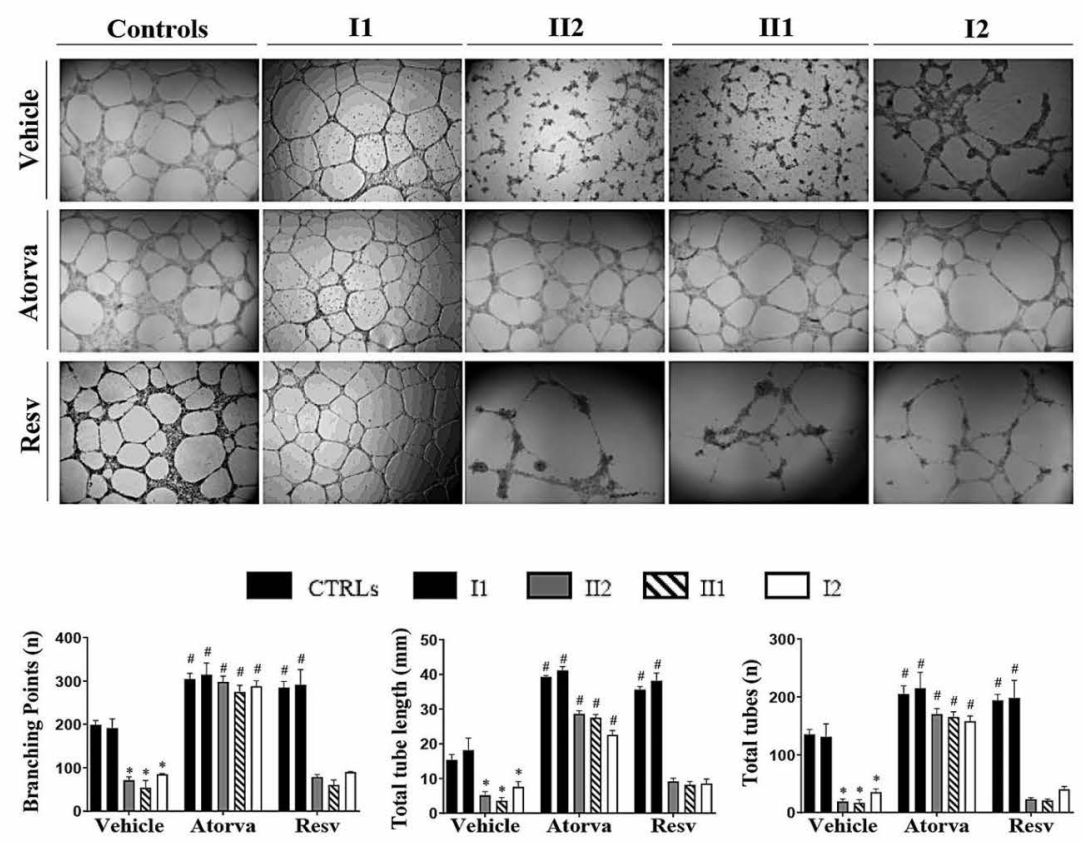

B

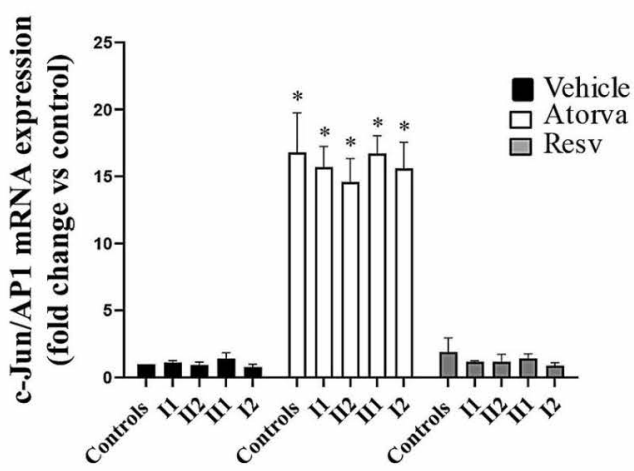

C

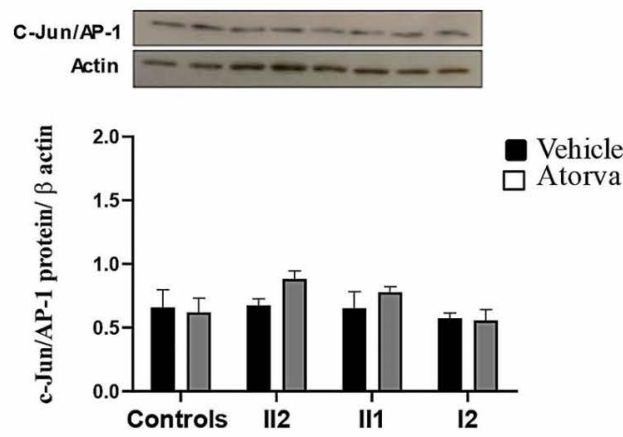

D C.Jun/AP-1

$$
\text { Actin }
$$

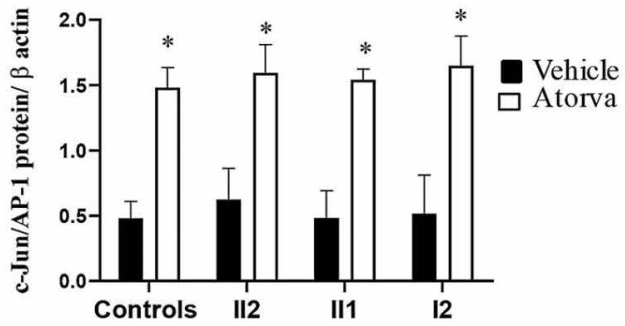

E

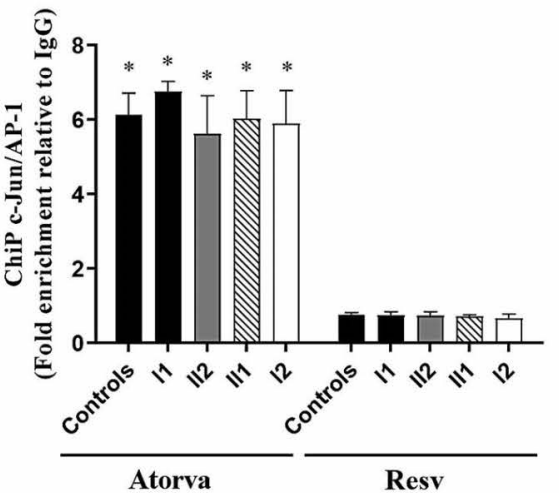

F

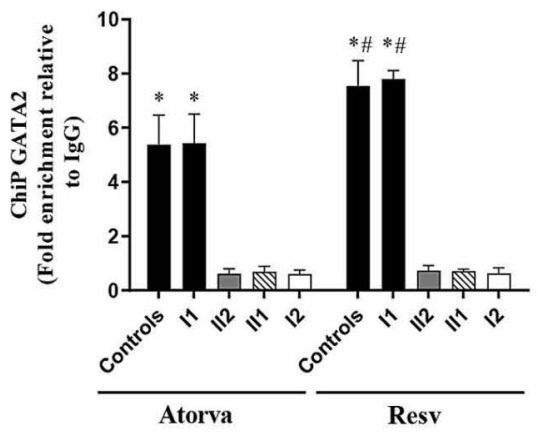


Figure 6 Atorvastatin restores eNOS expression and endothelial tube formation via AP1/c-Jun signaling. (A) Restored capillary tube formation in blood outgrowth endothelial cells (BOEC) from healthy controls (CTRL), the unaffected family member (I1) and the GATA2-deficient family members on matrigel-coated wells in comparison with healthy controls BOEC, after treatment with atorvastatin $(50 \mu \mathrm{M})$ for 24 hours. Angiogenesis was quantified with ImageJ (AngioTool64) software by measuring total tube length, tube number and branching points. Values are means \pm standard error of the mean (SEM) of 6 repeated measures ( ${ }^{*} P<0.001$ vs. controls, ${ }^{\sharp} P<0.001$ vs. Vehicle, two-way ANOVA followed by Tukey's multiple comparison test). Specimens were analyzed at room temperature by a Carl Zeiss Axio Observer.A1 microscope (Carl Zeiss Inc, Oberkochen, Germany) using a 2.5X Plan-Apochromat objective and images acquired using the AxioVision software (Carl Zeiss Inc). (B) Real time polymerase chain reaction (PCR) of c-Jun/AP-1 mRNA of BOEC from healthy controls, the unaffected family member (I1) and the GATA2-deficient family members after preincubation with atorvastatin $(50 \mu \mathrm{M})$ or resveratrol $(40 \mu \mathrm{M})$ for $24 \mathrm{~h}$. The expression of $c-J u n / A P-1$ mRNA is reported as fold change versus healthy control BOEC and normalized to a housekeeping mRNA (GAPDH). Values are means + SEM of 4 repeated measurements ( $* P<0.001$ vs. vehicle and resv, two-way ANOVA followed by Tukey's multiple comparison test). (C) Western blotting of c-Jun/AP-1 protein in BOEC from healthy controls and the GATA2-deficient family members after incubation with resveratrol $(40 \mu \mathrm{M})$ for $24 \mathrm{~h}$. $\beta$-actin was used as loading control. Optical densitometric analysis was performed using Image J software and results are expressed in arbitrary units. Values represent mean \pm SEM of 6 repeated measures (two-way ANOVA followed by Tukey's multiple comparison test). Results from the unaffected family member (I1) are shown in the Online Supplementary Figure S11. (D) Western blotting of c-Jun/AP-1 protein in BOEC from healthy controls and the GATA2-deficient family members after incubation with atorvastatin $(50 \mu \mathrm{M})$ for $24 \mathrm{~h}$. $\beta$-actin was used as loading control. Optical densitometric analysis was performed using ImageJ software and results are expressed in arbitrary units. Values represent mean \pm SEM of 6 repeated measures (*P<0.001 vs. vehicles, twoway ANOVA followed by Tukey's multiple comparison test). Results from the unaffected family member (I1) are shown in the Online Supplementary Figure S11. (E) Chromatin immunoprecipitation (ChiP) quantitative PCR (qPCR) using primers amplifying the endothelial nitric oxide synthase gene (eNOS) promoter regions that are recognized by AP-1. The figure shows the real time PCR of AP-1-bound chromatin of BOEC from healthy controls, the unaffected family member (I1) and the GATA2deficient family members in resting conditions and after treatment with the eNOS inducers atorvastatin and resveratrol. Values represent mean \pm SEM of 6 repeated measures ( $* P 0.001$ vs. resveratrol; two-way ANOVA followed by Dunnett's multiple comparison test). Data are shown as fold change over immunoglobulin g (IgG). (F) ChiP qPCR using primers amplifying the eNOS promoter regions that are recognized by GATA-2. The figure shows the real time PCR of GATA2-bound chromatin of BOEC from healthy controls, the unaffected family member (I1) and the GATA2-deficient family members in resting conditions and after treatment with the eNOS inducers atorvastatin and resveratrol. Values represent mean \pm SEM of 6 repeated measures $\left(* P<0.001\right.$ vs. GATA2-mutated patients, ${ }^{*} P<0.001$ vs. Atorva; two-way ANOVA followed by Dunnett's multiple comparison test). Data are shown as fold change over lgG.

BOEC but not from BOEC of patients with GATA2 deficiency (Figure $5 \mathrm{~B})$. These results were confirmed by measuring NO production in DAF-loaded BOEC (Figure 5C).

\section{The eNOS inducer atorvastatin restores angiogenesis in blood outgrowth endothelial cells from GATA2-deficient patients by upregulating the expression of AP-1/c-Jun}

Increased expression of eNOS induced by atorvastatin was associated with enhanced angiogenesis in both BOEC from healthy controls and from patients with GATA2 deficiency as shown by significantly increased tube number, branching points and tube length, while resveratrol was ineffective (Figure 6A). Treatment with atorvastatin or resveratrol did not increase GATA2 mRNA and protein expression either in patient or in control BOEC (Online Supplementary Figure S10A to C), including BOEC from the unaffected family member (I1) (Online Supplementary Figure S10D). On the other hand, atorvastatin increased the expression of another eNOS transcription factor, c-Jun/AP-1. In fact after 24 hours of incubation with atorvastatin BOEC from both healthy controls and GATA2-mutanted patients showed a significant increase of c-Jun/AP-1 mRNA expression, an effect not observed with resveratrol (Figure 6B). Western blotting confirmed a significant increase of c-Jun/AP-1 protein expression in BOEC from healthy controls and GATA2-deficient patients treated with atorvastatin, but not with resveratrol (Figure 6C and D). BOEC derived from the unaffected family member (I1) showed the same behavior of healthy control BOEC (Online Supplementary Figure S11). In order to confirm that increased eNOS expression after treatment with atorvastatin was due to enhanced c-Jun/AP-1 binding to DNA, we carried out qPCR of c-Jun/AP-1-bound chromatin which was significantly increased in both BOEC from GATA2-deficient patients and from healthy controls treated with atorvastatin, but not with resveratrol (Figure 6E). GATA2-bound chromatin was significantly increased in healthy control BOEC and the unaffected family member, compared to BOEC of GATA2-mutated patients (Figure $6 \mathrm{~F}$ ) both under resting conditions and after treatment with the eNOS inducers atorvastatin and resveratrol. Interestingly, preincubation with resveratrol increased GATA2-bound chromatin in healthy control BOEC significantly more than atorvastatin (Figure 6F). qPCR was confirmed by PCR followed by DNA electrophoresis (Online Supplementary Figure S12).

\section{The eNOS inducer resveratrol enhances eNOS expression by upregulating Runx1 and its interaction with GATA2}

Resveratrol, but not atorvastatin, enhanced Runx1 protein expression both in healthy control and GATA2-mutated BOEC (Figure 7A and B). However, despite increased Runx1 expression by resveratrol, GATA2-Runx1 binding was significantly lower in GATA2 deficiency patients compared to healthy controls (Figure 7C), including the unaffected family member (I1) (Online Supplementary Figure $S 13 A$ and $B$ ).

\section{Discussion}

Our study shows that i) GATA2 deficiency is associated with defective expression of eNOS by platelets and endothelial cells with impaired NO production, that ii) reduced eNOS expression in turn causes an altered angiogenic activity of endothelial cells, and that iii) treatment of endothelial cells from GATA2-deficient patients with the eNOS mRNA inducer atorvastatin restores eNOS expression, $\mathrm{NO}$ production and angiogenesis.

$\mathrm{NO}$ is a mediator released by platelets and endothelial cells which plays an important antithrombotic role by preventing almost all aspects of platelet activation, by displaying anti atherosclerotic effects, by reducing leukocyte adhesion and activation and by regulating vascular tone..$^{17-19,36} \mathrm{An}$ impaired effectiveness of $\mathrm{NO}$ or its rapid inactivation due to gene variants affecting guanylylcyclase or glutathione peroxidase have been shown to be responsible of an increased tendency to ischemic stroke and myocardial infarction ${ }^{37,38}$ and acquired endothelial dysfunction and NO deficiency are associated with enhanced risk of cardiovascular disease and venous thromboembolism..$^{20,21,36,39}$ We therefore hypothesize that the high incidence of thrombotic events among patients with GATA2 deficiency, so far largely unexplained, ${ }^{2}$ may depend to a great extent on the inability of their platelets and endothelial cells to produce NO. 
A
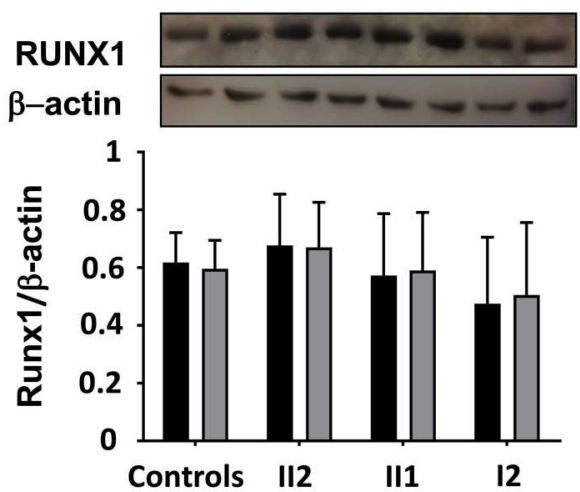

C

\section{IP:GATA2; WB:Runx1}
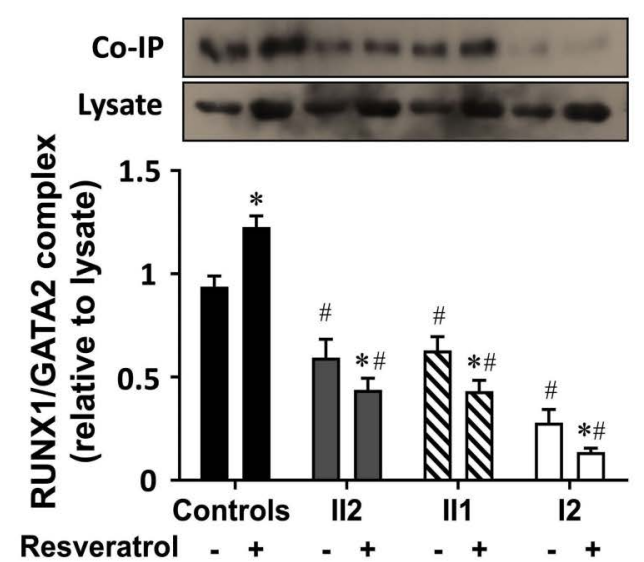

B

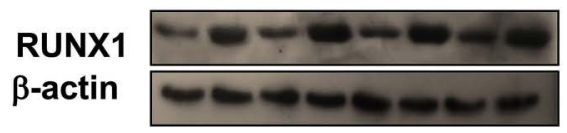

Vehicle Atorva

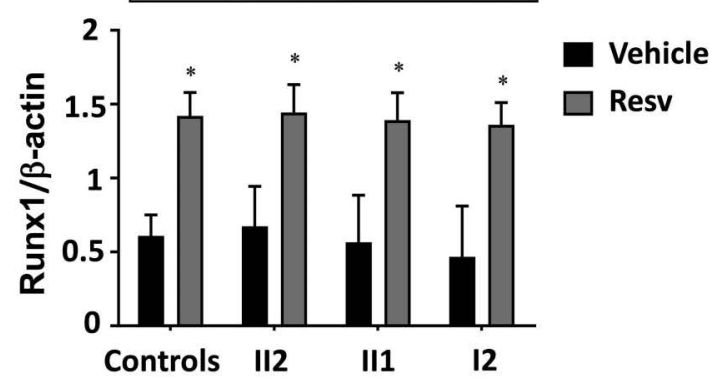

Figure 7. Resveratrol restores eNOS expression and endothelial tube formation via Runx1 signaling and its interaction with GATA2. (A) Western blotting of RUNX1 protein in blood outgrowth endothelial cells (BOEC) from healthy controls and from GATA2-mutated patients after incubation with atorvastatin $(50 \mu \mathrm{M})$ for 24 hours $(\mathrm{h})$. $\beta$-actin was used as loading control. Optical densitometric analysis was performed using ImageJ software and results are expressed in arbitrary units. Values represent mean \pm standard error of the mean (SEM) of 6 repeated measures (two-way ANOVA followed by Tukey's multiple comparison test). Results from the unaffected family member (I1) are shown in the Online Supplementary Figure S13A. (B) Western blotting of RUNX1 protein in BOEC from healthy controls and from GATA2-mutated patients after stimulus with resveratrol (40 $\mu \mathrm{M})$ for $24 \mathrm{~h}$. $\beta$-actin was used as loading control. Optical densitometric analysis was performed using ImageJ software and results are expressed in arbitrary units. Values represent mean \pm SEM of 6 repeated measures ( $* P<0.001$ vs. vehicle; two-way ANOVA followed by Tukey's multiple comparison test). Results from the unaffected family member (I1) are shown in the Online Supplementary Figure 13A. (C) Co-immunoprecipitation (Co-IP) of RUNX1-GATA2 complex in BOEC from healthy controls and from GATA2-mutated patients after incubation with resveratrol $(40 \mu \mathrm{M})$ for $24 \mathrm{~h}$. GATA2 protein was immunoprecipitated and western blotting was evaluated on RUNX1 protein. RUNX1 total lysate was used as INPUT for quantification. Optical densitometric analysis was performed using ImageJ software and results are expressed in arbitrary units. In patients with GATA2 deficiency the ratio Co-IP/lysates is reduced due to the increased Runx1 expression in lysates in response to resveratrol. Values represent mean \pm SEM of 6 repeated measures ( ${ }^{*} P<0.001$ vs. vehicle, ${ }^{*} P<0.001$ vs. controls, two-way ANOVA followed by Tukey's multiple comparison test). Results from the unaffected family member (I1) are shown in the Online Supplementary Figure S13B. eNOS: endothelial nitric oxide synthase gene.

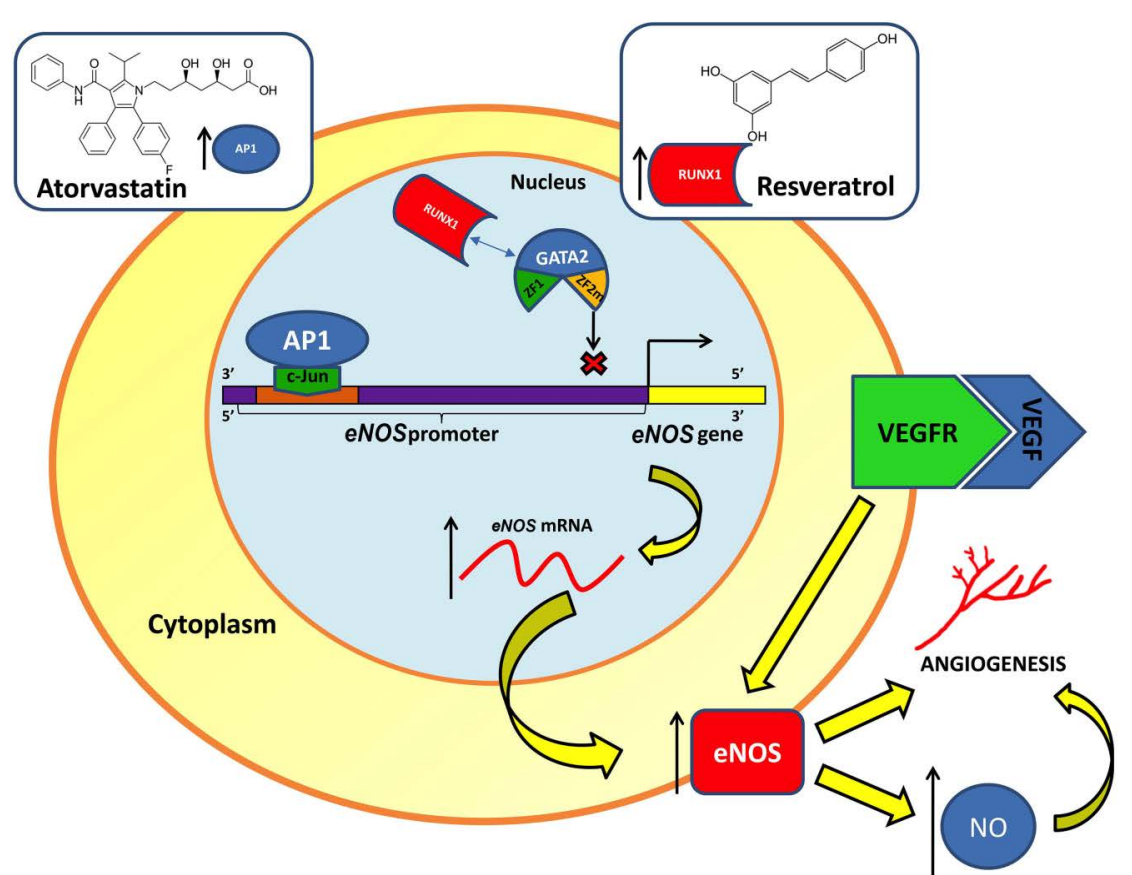

Figure 8. Mechanism of action of atorvastatin and resveratrol on eNOS expression and neoangiogenesis in patients with GATA2 deficiency. In R398W variant-carrying blood outgrowth endothelial cells (BOEC) the binding of GATA2 to DNA is reduced. Treatment with atorvastatin upregulates c-Jun/AP-1 which, when GATA2 is inactive, restores endothelial nitric oxide synthase gene (eNOS) mRNA and protein expression and thus nitric oxide (NO) production and neoangiogenesis. Resveratrol acts increasing RUNX1 expression and its binding to GATA2, therefore in GATA2 deficiency BOEC impaired eNOS transcription factor activity is not overcome. 
The role of GATA2 as a promoter of the eNOS gene in bovine aortic endothelial cells and in airway epithelial cells was previously reported, ${ }^{15,16}$ but so far no studies had analyzed the impact that GATA2 variants which are associated with hematopoietic impairment have on its transcription factor activity for eNOS and on eNOS expression and its function in human endothelial cells. Here we show that the R398W GATA2 variant, a germline mutation frequently found in patients with the GATA2 deficiency syndrome, ${ }^{40}$ impairs GATA2 binding to the eNOS gene in patientderived endothelial cells reducing the transcription of eNOS mRNA and consequently decreasing NO production. Concordantly, the silencing of GATA2 mRNA in BOEC from healthy controls, using a combination of siRNA targeting different sequences of the transcript, generated a NO production defect identical to that of GATA2-deficient patients.

The role of GATA2 in vascular development was also previously reported using human endothelial cells ${ }^{14}$ but no studies had explored angiogenesis in GATA2-deficient patients. Here we show that BOEC from patients with GATA2 deficiency display a striking impairment of endothelial tube formation and that this impairment is strictly dependent on $\mathrm{NO}$ insufficiency, in fact the supplementation of both GATA2-deficient BOEC and GATA2silenced control BOEC with exogenous NO restored a normal angiogenetic profile, while the treatment of control BOEC with the eNOS antagonist L-NIO generated an angiogenesis defect identical to that of GATA2-deficient BOEC. Several of the hematological and non-hematological manifestations of GATA2 deficiency have been associated with alterations in angiogenesis, like leukemia, solid organ tumors, lymphedema, venous thrombosis and stroke $e^{8,41,42}$ and GATA2-dependent pro- or anti-angiogenic microRNA regulation has been shown to have an important impact on endothelial biology and potentially on vascular disease. ${ }^{14}$ Therefore, our observation that defective eNOS provokes impaired angiogenesis in GATA2-deficient BOEC might be of relevance not only for the thrombotic but also for other clinical manifestations of the GATA2 deficiency syndrome.

Given the striking impairment of NO production by GATA2-deficient BOEC, we explored whether some known inducers of eNOS expression or activity might restore NO production. ${ }^{19,31,4,3,44}$ We show that treatment with the hydroxymethylglutaryl-CoenzymeA inhibitor atorvastatin, a known enhancer of eNOS mRNA expression, but not with the natural polyphenol resveratrol, an agent preventing oxidation-triggered eNOS uncoupling, largely restored eNOS expression and NO biosynthesis in GATA2deficient BOEC. Interestingly, atorvastatin almost completely restored also the angiogenic activity of $\mathrm{BOEC}$ from GATA2-deficient patients.

eNOS is an enzyme with a complex regulatory pattern, both at the transcriptional and post-transcriptional level, and sterol-regulatory cis-elements are present in the $5^{\prime}$ 'regulatory region of its gene suggesting that intracellular cholesterol levels are modulators of its expression. ${ }^{45,46}$ The cholesterol-lowering agent atorvastatin increases the expression of eNOS mRNA and protein by mechanisms not completely understood involving the blockade of Rho geranylgeranylation and the regulation of endoglin expression, and also enhances post-translationally eNOS activity by favoring its phosphorylation. ${ }^{194,750}$ It is thus likely that, despite the failure of the transcriptional function of GATA2, atorvastatin may restore eNOS mRNA transcription in BOEC from patients with GATA2 deficiency by stimulating the activity of other transcription factors involved in the regulation of eNOS expression. ${ }^{47}$ Conversely resveratrol, a polyphenol with antioxidant effects and multiple beneficial activities on vascular function, ${ }^{43}$ enhanced eNOS expression in control BOEC but did not restore it in GATA2-deficient BOEC. Resveratrol upregulates eNOS expression acting through transcriptional and posttranscriptional (stabilization of mRNA) mechanisms. ${ }^{33}$ The discrepancy between the effects of atorvastatin and resveratrol in GATA2 deficiency BOEC may be due to their effect at different positions of the promoter sequence. Atorvastatin increases eNOS mRNA by enhancing the activity of transcription factors that bind the eNOS promoter in the $5^{\prime}$ region, ${ }^{32}$ including the transcription factor c-Jun/AP- $1^{15}$ which was in fact significantly increased by this drug in both healthy control and GATA2-mutated BOEC. Differently, resveratrol enhances the activity of transcription factors that bind the eNOS promoter in the proximal $263 \mathrm{bp}$ region ${ }^{33}$ that involves the GATA2 binding site (254-279 bp)..$^{16}$ Interestingly, we found that resveratrol enhances eNOS expression in normal endothelial cells by increasing RUNX1. RUNX1 displays its transcription factor activity function by interacting with GATA2 $^{51,52}$ and in the absence of RUNX1, GATA2 may rescue its transcriptional activity supporting hematopoiesis, which explains why the knockout of GATA2 reduces survival of RUNX1 ${ }^{*}$ zebrafish. ${ }^{53}$ The dependency of RUNX1 transcription factor activity on GATA2 to display its function explains why a ZNF2 GATA2 mutation, reducing GATA2 binding to DNA, prevents eNOS upregulation despite increased RUNX1 expression by resveratrol (Figure 8).

Statins significantly reduce the risk of ischemic cardioand cerebro-vascular events and of venous thromboembolism, in part due to their ability to restore NO production, ${ }^{54,56}$ with little side effects and no hemorrhagic risk. Based on our results, the preventive effect of atorvastatin on thrombotic events in patients with GATA2 deficiency, and possibly its beneficial effects on other clinical manifestations of the syndrome related to deranged angiogenesis, should be explored in an ad hoc designed clinical trial. It should be considered that our results were obtained from studies of a single family with a specific in variant of the GATA2 gene and, thus, given the variant heterogeneity on GATA2 deficiencies, they might not be relevant for all cases of GATA2 deficiency.

Our study reports the first observation, to our knowledge, of a germline gene variant-induced alteration of eNOS expression in humans and unravels the cause of altered angiogenesis in GATA2-deficient patients, suggesting that this may represent an important thrombogenic mechanism in these patients. We also identified a therapeutic option, through atorvastatin, able to restore eNOS expression and angiogenesis in GATA2 deficiency which deserves to be tested in vivo.

\section{Disclosures \\ No conflicts of interest to disclose.}

\section{Contributions}

$G P, E P, G G, E F, L B, V B, F P, F M$ and $C M$ a performed experiments, analyzed and interpreted data; CMe and PG designed and supervised the study; CM contributed the patient for the study; $G P$ and $P G$ wrote the manuscript; all authors critically revised the manuscript. 


\section{Acknowledgments}

We thank M.P. Martelli for help with confocal microscopy studies.

\section{Fundings}

This study was supported by AIRC $5 \times 1000$, MYNERVA project, \#21267 (Myeloid Neoplasms Research Venture Airc,
http://Www.progettomynerva.it/). EF and $L B$ were supported by a fellowship from Fondazione Umberto Veronesi.

\section{Data sharing statement}

All data generated and analyzed during this study are included in this published article and its additional file.

\section{References}

1. Bresnick EH, Katsumura KR, Lee HY, Johnson KD, Perkins AS. Master regulatory GATA transcription factors: mechanistic principles and emerging links to hematologic malignancies. Nucleic Acids Res. 2012;40(13):5819-5831.

2. McReynolds LJ, Calvo KR, Holland SM. Germline GATA2 mutation and bone marrow failure. Hematol Oncol Clin North Am. 2018;32(4):713-728.

3. Collin M, Dickinson R, Bigley V. Haematopoietic and immune defects associated with GATA2 mutation. Br J Haematol. 2015;169(2):173-187.

4. Hsu AP, Sampaio EP, Khan J, et al. Mutations in GATA2 are associated with the autosomal dominant and sporadic monocytopenia and mycobacterial infection (MonoMAC) syndrome. Blood. 2011;118(10):2653-2655.

5. Dickinson RE, Griffin H, Bigley V, et al. Exome sequencing identifies GATA-2 mutation as the cause of dendritic cell, monocyte, B and NK lymphoid deficiency. Blood. 2011;118(10):2656-2658.

6. Hahn CN, Chong CE, Carmichael CL, et al. Heritable GATA2 mutations associated with familial myelodysplastic syndrome and acute myeloid leukemia. Nat Genet. 2011;43(10):1012-1017.

7. Ostergaard P, Simpson MA, Connell FC, et al. Mutations in GATA2 cause primary lymphedema associated with a predisposition to acute myeloid leukemia (Emberger syndrome). Nat Genet. 2011;43(10):929931.

8. Spinner MA, Sanchez LA, Hsu AP, et al. GATA2 deficiency: a protean disorder of hematopoiesis, lymphatics, and immunity. Blood. 2014;123(6):809-821.

9. Crispino JD, Horwitz MS. GATA factor mutations in hematologic disease. Blood. 2017;129(15):2103-2110.

10. Zolton JR, Parikh TP, Hickstein DD, et al. Oocyte cryopreservation for women with GATA2 deficiency. J Assist Reprod Genet. 2018;35(7):1201-1207.

11. Berry D, Fekrat S. Central retinal vein occlusion in GATA2 deficiency. Retin Cases Brief Rep. 2019;13(2):181-184.

12. Johnson KD, Hsu AP, Ryu MJ, et al. Cis-element mutated in GATA2-dependent immunodeficiency governs hematopoiesis and vascular integrity. J Clin Invest. 2012;122(10):3692-3704.

13. Linneman AK, O'Geen H, Keles S, Farnham PJ, Bresnick EH. Genetic framework for GATA factor function in vascular biology. Proc Natl Acad Sci USA. 2011;108(33): 13641-13646.

14. Hartmann D, Fiedler J, Sonnenschein K, et al. MicroRNA-based therapy of GATA2deficient vascular disease. Circulation. 2016:134(24):1973-1990.

15. Zhang R, Min W, Sessa WC. Functional analysis of the human endothelial nitric oxide synthase promoter. Sp1 and GATA factors are necessary for basal transcription in endothelial cells. J Biol Chem. 1995;270(25):15320-15326.

16. German Z, Chambliss KL, Pace MC, Arnet UA, Lowenstein CJ, Shaul PW. Molecular basis of cell-specific endothelial nitric-oxide synthase expression in airway epithelium. J Biol Chem. 2000;275(11):8183-8189.

17. Reichenbach G, Momi S, Gresele P. Nitric oxide and its antithrombotic action in the cardiovascular system. Curr Drug Targets Cardiovasc Haematol Disord. 2005;5(1): 65-74.

18. Tziros C, Freedman JE. The many antithrombotic actions of nitric oxide. Curr Drug Targets. 2006;7(10):1243-1251.

19. Gresele P, Momi S, Guglielmini G. Nitric oxide-enhancing or -releasing agents as antithrombotic drugs. Biochem Pharmacol. 2019;166:300-312.

20. Migliacci R, Becattini C, Pesavento R, et al Endothelial dysfunction in patients with spontaneous venous thromboembolism. Haematologica. 2007:92(6):812-818.

21. Momi S, Falcinelli E, Evangelista S, Gresele P. Antithrombotic and in vivo antiplatelet activity of Nebivolol are mediated by increased platelet-derived nitric oxide. Arterioscler Thromb Vasc Biol. 2014;34(4) 820-829.

22. Martin-Ramirez J, Hofman M, Van Den Biggelaar M, Hebbel RP, Voorberg J. Establishment of outgrowth endothelial cells from peripheral blood. Nat Protocols. 2012;7(9):1709-1715

23. Cecchetti L, Tolley ND, Michetti N, Bury L, Weyrich AS, Gresele P. Megakaryocytes differentially sort mRNAs for matrix metalloproteinases and their inhibitors into platelets: a mechanism for regulating synthetic events. Blood. 2011;118(7):19031911.

24. Mikkelsen TS, Ku M, Jaffe DB, et al. Genome-wide maps of chromatin state in pluripotent and lineage-committed cells. Nature. 2007;448(7153):553-560.

25. He S, Wang F, Yang L, et al. Expression of DNMT1 and DNMT3a are regulated by GLI1 in human pancreatic cancer. PLoS One. 2011;6(11):e27684.

26. Sebastiano M, Momi S, Falcinelli E, Bury L Hoylaerts MF, Gresele P. A novel mechanism regulating human platelet activation by MMP-2-mediated PAR1 biased signaling. Blood. 2017;129(7),883-895.

27. Amison RT, Momi S, Morris A, et al. RhoA signaling through platelet $\mathrm{P}_{2} \mathrm{Y}_{1}$ receptor controls leukocyte recruitment in allergic mice. J Allergy Clin Immunol. 2015; 135(2):528-538.

28. Bury L, Malara A, Momi S, Petito E, Balduini A, Gresele P. Mechanisms of thrombocytopenia in platelet-type von Willebrand disease. Haematologica. 2019;104(7):1473-1481

29. Balaguer S, Diaz L, Gomes A, et al. RealTime cytometric assay of nitric oxide and superoxide interaction in peripheral blood monocytes: a no-wash, no-lyse kinetic method. Cytometry Part B Clinical
Cytometry. 2015;92(3):211-217

30. Cozzi MR, Guglielmini G, Battiston M, et al. Visualization of nitric oxide production by individual platelets during adhesion in flowing blood. Blood. 2015;125(4):697-705

31. Gresele P, Pignatelli P, Guglielmini G, et al. Resveratrol, at concentrations attainable with moderate wine consumption, stimulates human platelet nitric oxide production. J Nutr. 2008;138(9):1602-1608.

32. Mashimo Y, Ishikawa T, Numakura M, Kinoshita M, Teramoto T. Critical promoter region for statin-induced human endothelial nitric oxide synthase (eNOS) transcription in EA.hy926 cells. Atheroscler Thromb. 2013; 20(4):321-329.

33. Wallerath T, Deckert G, Ternes T, et al. Resveratrol, a polyphenolic phytoalexin present in red wine, enhances expression and activity of endothelial nitric oxide synthase. Circulation. 2002;106(13):1652-1658.

34. Khoo CP, Micklem K, Watt SM. A comparison of methods for quantifying angiogenesis in the matrigel assay in vitro. Tissue Eng Part C Methods. 2011;17(9):895-906.

35. Frye M, Taddei A, Dierkes C, et al. Matrix stiffness controls lymphatic vessel formation through regulation of a GATA2-dependent transcriptional program. Nat Commun. 2018;9(1):1511.

36. Gresele P, Momi S, Migliacci R Endothelium, venous thromboembolism and ischaemic cardiovascular events. Thromb Haemost. 2010;103(1):56-61

37. Freedman JE, Loscalzo J, Benoit SE, Valer CR, Barnard MR, Michelson AD. Decreased platelet inhibition by nitric oxide in two brothers with a history of arterial thrombosis. J Clin Invest 1996;97(4);979-987

38. Erdmann J, Stark K, Esslinger UB, et al Dysfunctional nitric oxide signalling increases risk of myocardial infarction. Nature. 2013;504(7480)432-436.

39. Suzuki H, Matsuzawa Y, Konishi M, et al. Utility of noninvasive endothelial function test for prediction of deep vein thrombosis after total hip or knee arthroplasty. Circ J. 2014;78(7):1723-1732.

40. Chong CE, Venugopal P, Stokes PH, et al Differential effects on gene transcription and hematopoietic differentiation correlate with GATA2 mutant disease phenotypes. Leukemia. 2018;32(1):194-202.

41. Rodríguez-Caso L, Reyes-Palomares A, Sánchez-Jiménez F, Quesada AR, Medina MÁ. What is known on angiogenesis-related rare diseases? A systematic review of literature. J Cell Mol Med. 2012;16(12):28722893.

42. Sun LL, Li WD, Lei FR, Li XO. The regulatory role of microRNAs in angiogenesisrelated diseases. I Cell Mol Med. 2018:22(10):4568-4587.

43. Gresele P, Cerletti C, Guglielmini G, Pignatelli P, De Gaetano G, Violi F. Effects of resveratrol and other wine polyphenols on vascular function: an update. J Nutr Biochem. 2011;22(3):201-211.

44. Forstermann U, Li H. Therapeutic effect of 
enhancing endothelial nitric oxide syntase (eNOS) expression and preventing eNOS uncoupling. Br J Pharmacol. 2011;164(2): 213-223.

45. Marsden PA, Heng HH, Scherer SW, et al. Structure and chromosomal localization of the human constitutive endothelial nitric oxide synthase gene. I Biol Chem. 1993;268(23):17478-17488.

46. Robinson LJ, Weremowicz S, Morton CC, Michel T. Isolation and chromosomal localization of the human endothelial nitric oxide synthase (NOS3) gene. Genomics. 1994;19(2):350-357.

47. Garcia V, Sessa WC. Endothelial NOS: perspective and recent developments. Br J Pharmacol. 2019;176(2):189-196.

48. Zemankova L, Varejckova M, Dolezalova E, et al. Atorvastatin-induced endothelial nitric oxide synthase expression in endothelial cells is mediated by endoglin. J Physiol Pharmacol. 2015;66(3):403-413.

49. Laufs U, La Fata V, Plutzky J, Liao JK Upregulation of endothelial nitric oxide synthase by HMG CoA reductase inhibitors. Circulation. 1998;97(12):11291135.

50. Momi S, Monopoli A, Alberti PF, et al. Nitric oxide enhances the anti-inflammatory and anti-atherogenic activity of atorvastatin in a mouse model of accelerated atherosclerosis. Cardiovasc Res. 2012;94(3): 428-438.

51. Kaur S, Rawal P, Siddiqui H, et al. Increased expression of RUNX1 in liver correlates with NASH activity score in patients with non-alcoholic steatohepatitis (NASH). Cells. 2019;8(10):1277.

52. Wilson NK, Foster SD, Wang $\mathrm{X}$, et al. Combinatorial transcriptional control in blood stem/progenitor cells: genome-wide analysis of ten major transcriptional regulators. Cell Stem Cell. 2010;7(4):532-544

53. Bresciani E, Carrington B, Kwon Kim EM et al. GATA2 is required for the runx1-independent hematopoiesis in Zebrafish. Blood 2019; 134 (Suppl 1):2463

54. Blum A, Shamburek R. The pleiotropic effects of statins on endothelial function, vascular inflammation, immunomodulation and thrombogenesis. Atherosclerosis. 2009;203(2);325-330

55. Oesterle A, Laufs U, Liao JK. Pleiotropic effects of statins on the cardiovascular system. Circ Res. 2017;120(1):229-243.

56. Paciullo F, Momi S, Gresele P. PCSK9 in haemostasis and thrombosis: possible pleiotropic effects of PCSK9 inhibitors in cardiovascular prevention. Thromb Haemost. 2019;119(3):359-367. 